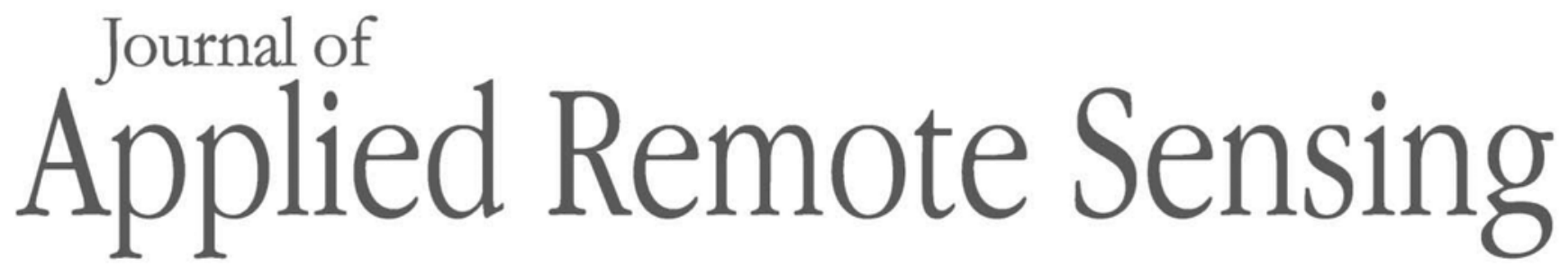

RemoteSensing.SPIEDigitalLibrary.org

\title{
Study of sea-surface slope distribution and its effect on radar backscatter based on Global Precipitation Measurement Ku-band precipitation radar measurements
}

\author{
Qiushuang Yan \\ Jie Zhang \\ Chenqing Fan \\ Jing Wang \\ Junmin Meng
}

Qiushuang Yan, Jie Zhang, Chenqing Fan, Jing Wang, Junmin Meng, "Study of sea-surface slope distribution and its effect on radar backscatter based on Global Precipitation Measurement Ku- 


\title{
Study of sea-surface slope distribution and its effect on radar backscatter based on Global Precipitation Measurement Ku-band precipitation radar measurements
}

\author{
Qiushuang Yan, ${ }^{\text {a,b }}$ Jie Zhang, ${ }^{\mathrm{b}}$ Chenqing Fan, ${ }^{\mathrm{b}, *}$ Jing Wang, ${ }^{\mathrm{a}}$ and \\ Junmin Meng ${ }^{\mathrm{b}}$ \\ ${ }^{a}$ Ocean University of China, College of Information and Engineering, Qingdao City, \\ Shandong Province, China \\ ${ }^{\mathrm{b}}$ State Oceanic Administration, First Institute of Oceanography, Qingdao City, \\ Shandong Province, China
}

\begin{abstract}
The collocated normalized radar backscattering cross-section measurements from the Global Precipitation Measurement (GPM) Ku-band precipitation radar (KuPR) and the winds from the moored buoys are used to study the effect of different sea-surface slope probability density functions (PDFs), including the Gaussian PDF, the Gram-Charlier PDF, and the Liu PDF, on the geometrical optics (GO) model predictions of the radar backscatter at low incidence angles $(0 \mathrm{deg}$ to $18 \mathrm{deg})$ at different sea states. First, the peakedness coefficient in the Liu distribution is determined using the collocations at the normal incidence angle, and the results indicate that the peakedness coefficient is a nonlinear function of the wind speed. Then, the performance of the modified Liu distribution, i.e., Liu distribution using the obtained peakedness coefficient estimate; the Gaussian distribution; and the Gram-Charlier distribution is analyzed. The results show that the GO model predictions with the modified Liu distribution agree best with the KuPR measurements, followed by the predictions with the Gaussian distribution, while the predictions with the Gram-Charlier distribution have larger differences as the total or the slick filtered, not the radar filtered, probability density is included in the distribution. The bestperforming distribution changes with incidence angle and changes with wind speed. (c) The Authors. Published by SPIE under a Creative Commons Attribution 3.0 Unported License. Distribution or reproduction of this work in whole or in part requires full attribution of the original publication, including its DOI. [DOI: 10.1117/1.JRS.12.016006]
\end{abstract}

Keywords: Global Precipitation Measurement Ku-band precipitation radar cross-section measurements; sea-surface slope distribution; geometrical optics model; peakedness coefficient determination; performance comparison.

Paper 170693 received Aug. 3, 2017; accepted for publication Dec. 8, 2017; published online Jan. 4, 2018.

\section{Introduction}

The radar backscatter from the ocean surface is closely related to the surface slope distribution, which is a statistic that can be used to quantitatively describe the ocean surface roughness. For example, quasispecular reflection is thought to dominate the microwave backscattering at small (near-nadir) incidence angles, and, based on the geometrical optics (GO) approach, the radar cross-section due to quasispecular reflection is proportional to the probability density function (PDF) of the sea-surface slopes. ${ }^{1-3}$ The simple analytic formulation is written as follows:

$$
\sigma_{0}=\pi \sec ^{4} \theta p\left(\zeta_{x}, \zeta_{y}\right)|R(0)|^{2},
$$

where $\sigma_{0}$ is the normalized radar cross-section or the backscatter coefficient in natural units (not in decibels), $\theta$ is the radar incidence angle, $R(0)$ is the Fresnel reflection coefficient for normal

*Address all correspondence to: Chenqing Fan, E-mail: fanchenqing @fio.org.cn 
incidence, and $p\left(\zeta_{x}, \zeta_{y}\right)$ is the joint PDF of the sea-surface slopes. Here, $\zeta$ is the surface elevation and $\zeta_{x}=\partial \zeta / \partial x$ and $\zeta_{y}=\partial \zeta / \partial y$ are the surface slopes in the upwind and crosswind directions, respectively. The slope PDF is evaluated at the specular points, which correspond to $\zeta_{x}=\tan \theta \cos \phi_{w}$ and $\zeta_{y}=\tan \theta \sin \phi_{w}$ and $\phi_{w}$ is the angle between the wind direction and the horizontal antenna direction. In using Eq. (1) to predict the radar backscatter from the ocean surface, only the portion of the total probability density contributed by ocean waves with lengths greater than the radar wave length is included. ${ }^{2}$

In linear wave theory, the sea-surface slopes are assumed to be Gaussian according to the central limit theorem. For a nonisotropic Gaussian rough surface, the following is true:

$$
p\left(\zeta_{x}, \zeta_{y}\right)=\frac{1}{2 \pi s_{u} s_{c}} \exp \left[-\frac{1}{2}\left(\frac{\zeta_{x}^{2}}{s_{u}^{2}}+\frac{\zeta_{y}^{2}}{s_{c}^{2}}\right)\right]
$$

where $s_{u}$ and $s_{c}$ are the root mean square (RMS) slopes for the upwind and crosswind directions, respectively. Previous studies have found that when the Gaussian PDF was used, the model prediction was in good agreement with the measured data at the normal incidence angle, but it was in considerable disagreement when extended to angles away from normal incidence, implying that the slope distribution in the open ocean is not Gaussian.,

Cox and Munk ${ }^{5}$ found that the sea-slope PDF was well fitted by a Gram-Charlier expansion through the statistical analysis of the observed slopes from airborne photographs of sun glint. Compared with the Gaussian distribution, the Gram-Charlier distribution includes two additional factors: peakedness and skewness. The Gram-Charlier distribution has a higher accuracy for the range of the small slopes, which are defined as less than 2.5 times the RMS slope. ${ }^{5,6}$ The Gram-Charlier PDF is expressed as follows:

$$
\begin{aligned}
p\left(\zeta_{x}, \zeta_{y}\right)= & \frac{1}{2 \pi s_{u} s_{c}} \exp \left[-\frac{1}{2}\left(\frac{\zeta_{x}^{2}}{s_{u}^{2}}+\frac{\zeta_{y}^{2}}{s_{c}^{2}}\right)\right] \\
& \times\left[\begin{array}{l}
1-\frac{1}{2} c_{12} \frac{\zeta_{x}}{S_{u}}\left(\frac{\zeta_{y}^{2}}{s_{c}^{2}}-1\right)-\frac{1}{6} c_{30}\left(\frac{\zeta_{x}^{3}}{s_{u}^{3}}-3 \frac{\zeta_{x}}{s_{u}}\right)+\frac{1}{24} c_{40}\left(\frac{\zeta_{x}^{4}}{s_{u}^{4}}-6 \frac{\zeta_{x}^{2}}{s_{u}^{2}}+3\right) \\
+\frac{1}{4} c_{22}\left(\frac{\zeta_{x}^{2}}{s_{u}^{2}}-1\right)\left(\frac{\zeta_{y}^{2}}{s_{c}^{2}}-1\right)+\frac{1}{24} c_{04}\left(\frac{\zeta_{y}^{4}}{s_{c}^{4}}-6 \frac{\zeta_{y}^{2}}{s_{c}^{2}}+3\right)
\end{array}\right],
\end{aligned}
$$

where $c_{12}$ and $c_{30}$ are the skewness coefficients and $c_{40}, c_{22}$, and $c_{04}$ are the peakedness coefficients. Cox and Munk ${ }^{5}$ determined these five coefficients as well as $s_{u}^{2}$ and $s_{c}^{2}$ as functions of wind speed for clean and slick water based on the sun glitter observations of the sea surface. Bréon and Heriot $^{7}$ performed a similar analysis using measurements of the ocean reflectance from the polarization and directionality of the earth reflectances multidirectional radiometer and the ocean surface wind from the National Aeronautics and Space Administration (NASA) scatterometer. However, these functions may not be appropriate for the prediction of radar backscatter because they define total slopes or slick filtered slopes, not radar filtered slopes. The Gram-Charlier PDF becomes negative when the slope is large. For the range of large slopes, neither the Gaussian nor the Gram-Charlier distributions work. Liu et al. ${ }^{4}$ derived a more appropriate PDF:

$$
p\left(\zeta_{x}, \zeta_{y}\right)=\frac{n}{2 \pi(n-1) s_{u} s_{c}} \times\left[1+\frac{\zeta_{x}^{2}}{(n-1) s_{u}^{2}}+\frac{\zeta_{y}^{2}}{(n-1) s_{c}^{2}}\right]^{-(n+2) / 2}+\text { skewness }
$$

where $n$ is the peakedness coefficient. The Liu PDF is an improvement over the Gram-Charlier distribution. It fits the Gram-Charlier distribution in the range of small slopes and works well in the full range of surface slopes. ${ }^{4}$ However, the application of the Liu distribution is limited as the explicit expressions to determine the peakedness and skewness coefficients are not given in previous studies. The skewness can be ignored because of its very small order. ${ }^{6}$ In this paper, a reasonable estimate of the peakedness coefficient in the Liu distribution is obtained using the Global Precipitation Measurement (GPM) Ku-band precipitation radar (KuPR) cross-section data and buoy wind speed data. Then, the performance of the Liu distribution using the obtained peakedness coefficient estimate (named the modified Liu distribution, hereafter), the Gaussian 
Yan et al.: Study of sea-surface slope distribution and its effect on radar backscatter...

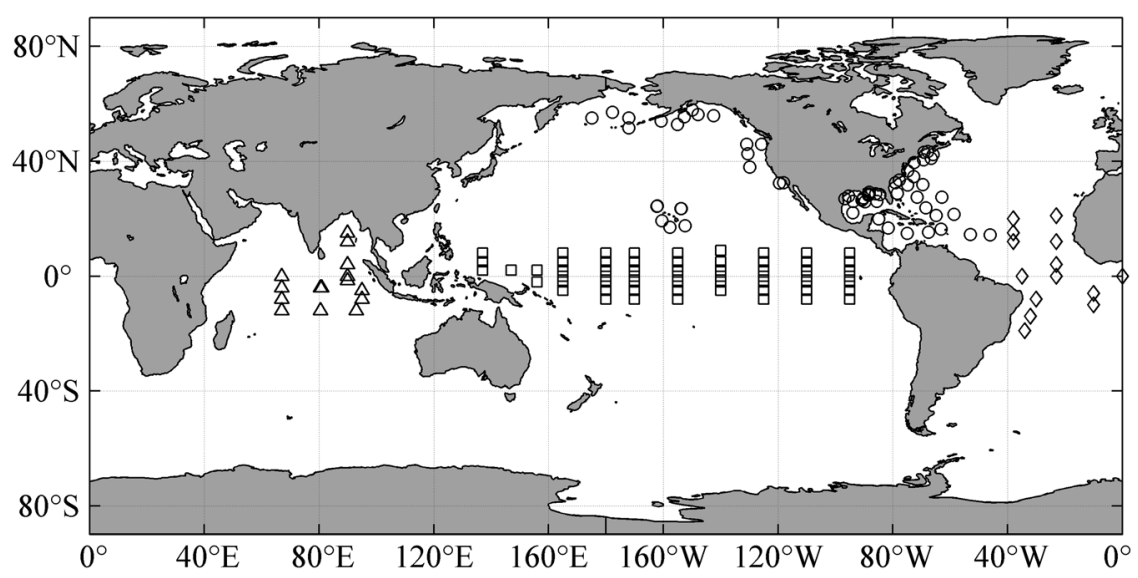

Fig. 1 Location of the NDBC (circle), TAO (square), RAMA (triangle), and PIRATA (diamond) buoys used in the present study.

distribution, and the Gram-Charlier distribution in predicting the radar backscatter at low incidence angles at different sea states is analyzed.

\section{Data}

\subsection{GPM Ku-Band Precipitation Radar Cross-Section Data}

GPM is an international satellite mission to measure global precipitation using one GPM core satellite and other subsatellites, and it is a mission similar to but broader than the Tropical Rainfall Measuring Mission (TRMM). The NASA and the Japanese Aerospace Exploration Agency (JAXA) launched the GPM core observatory satellite on February 27, 2014, carrying two instruments, including the dual-frequency precipitation radar (DPR) developed by Japan and the GPM microwave imager developed by the US. The GPM core satellite is able to observe not only the tropical zone but also mid- to high-latitude areas and can capture daily changes in precipitation at a $65 \mathrm{deg}$ inclined nonsun-synchronous orbit.

The DPR comprises a KuPR and a Ka-band precipitation radar. The KuPR, an updated version of the highly successful unit on the TRMM satellite, is a pulsed radar with an active electronically scanned phase array antenna that operates at $13.6 \mathrm{GHz}$ and a horizontal polarization that scans a plane normal to the flight direction over a $245-\mathrm{km}$ ground swath. The scan angle of the KuPR radar is relatively low, ranging from 0 deg to $18 \mathrm{deg}$ on each side of the satellite flight track. It can measure the ocean surface radar backscatter in 49 cross-track bins within one scan, which are separated by $\sim 0.71 \mathrm{deg}$ in incidence angle, corresponding to a spatial resolution of $5.2 \mathrm{~km}$.

The data product used herein is the GPM KuPR level 2 standard product (version 4) obtained from the globe portal system (G-Portal) of JAXA. It contains the normalized radar backscattering cross-section measurements, incidence angle information, standard quality flags, and a rain/norain flag for each measurement cell. The KuPR level 2 product for the 1-year period of 2015 is used for the data analysis. Only the high-quality measurements obtained over the ocean under rain-free conditions are selected.

\subsection{Buoy Wind Data}

Surface wind observations from buoys operated by different entities are used as a common reference to quantify the wind dependence of the KuPR backscatter cross-section in this study. The buoys included 60 moored buoys operated by the National Data Buoy Center (NDBC), 60 buoys of the Tropical Atmosphere Ocean (TAO) array in the Pacific Ocean, 15 buoys of the Pilot Research Moored Array in the tropical Atlantic (PIRATA), and 15 buoys of the Research 
Moored Array for African-Asian-Australian Monsoon Analysis and Prediction (RAMA). Figure 1 shows the locations of these buoys, all of which are located more than $50 \mathrm{~km}$ away from land. The NDBC wind is obtained by averaging the time series of the instantaneous wind samples taken over an 8-min period, and the standard measurements are reported hourly. The accuracy of the NDBC wind speed is determined to be $0.55 \mathrm{~m} / \mathrm{s}$ through comparisons between duplicate sensors on the same buoy, and the accuracy of the wind direction is assumed to be 9.26 deg based on adjacent buoy comparisons. ${ }^{8}$ The TAO, PIRATA, and RAMA winds are sampled over a 2-min period and are stored hourly or at every $10 \mathrm{~min}$. The accuracies of the winds measured at different stations with different types of wind sensors vary. Typically, the root mean square errors (RMSEs) of wind speed and direction are assumed to be $\sim 0.3 \mathrm{~m} / \mathrm{s}$ (or $3 \%$ ) and $5 \mathrm{deg}$, respectively, based on comparisons among a group of sensors. ${ }^{9,10}$ The wind measurements sampled at the anemometer height (normally 2 to $5 \mathrm{~m}$ ) are converted to $10-\mathrm{m}$ neutral winds after eliminating low-quality measurements. Then, the KuPR measurements are collocated with the buoy wind data with the following criteria: time separations within $1 \mathrm{~h}$ and spatial separations of less than $50 \mathrm{~km}$. The collocation procedure applied to the 1 year of data yielded more than 25,000 KuPR cross-section and buoy wind pairs at each KuPR incidence angle.

\section{Determination of the Peakedness Coefficient of the Liu Distribution}

\subsection{Determination Approach}

For nadir-looking, that is, the incidence angle being zero, we obtain the following from Eqs. (1) and (4):

$$
\sigma_{0}(0)=\frac{n}{n-1} \frac{|R(0)|^{2}}{2 s_{u} s_{c}}
$$

It can be seen that the ocean surface microwave backscatter at normal incidence angle is directly proportional to the normal incidence Fresnel reflection coefficient and is inversely proportional to the peakedness coefficient and the radar filtered RMS slopes. Thus, it is rather simple to determine the peakedness coefficient using the collocations at normal incidence. The $\sigma_{0}$ is often expressed in decibels, such that $\sigma_{0}^{\mathrm{dB}}=10 \log _{10}\left(\sigma_{0}\right)$. Then, Eq. (5) becomes

$$
\sigma_{0}^{\mathrm{dB}}(0)=10 \log _{10}\left(|R(0)|^{2}\right)-10 \log _{10}\left(2 s_{u} s_{c}\right)+10 \log _{10}\left(\frac{n}{n-1}\right) .
$$

For a smooth surface, the normal incidence Fresnel reflection coefficient $R(0)$ is calculated from the following:

$$
R(0)=(1-\sqrt{\varepsilon}) /(1+\sqrt{\varepsilon}),
$$

where $\varepsilon$ is the relative dielectric constant, which is obtained through the double-Debye dielectric model (D3M) developed by William Ellison. ${ }^{11}$ For the Ku-band (13.6 GHZ), $|R(0)|^{2}$ is $\sim 0.61$ on average over the open ocean, and $10 \log _{10}\left(|R(0)|^{2}\right)=-2.1 \mathrm{~dB}$. However, there is a discrepancy between the effective reflection coefficient and Fresnel reflectivity. $\mathrm{Wu}^{12}$ suggested that a $2.1 \mathrm{~dB}$ downward shift is needed for the Ku-band $10 \log _{10}\left(|R(0)|^{2}\right)$ calibration. The decay is probably caused by the surface tilting effect that modifies the local incident angle and hence reduces the backscattering intensity. ${ }^{13}$ Then, Eq. (6) becomes the following:

$$
\sigma_{0}^{\mathrm{dB}}(0)=-4.2(\mathrm{~dB})-10 \log _{10}\left(2 s_{u} s_{c}\right)-10 \log _{10}\left(1-\frac{1}{n}\right) .
$$

From Eq. (8), it can be seen that the values of the radar backscatter cross-section at normal incidence $\left[\sigma_{0}^{\mathrm{dB}}(0)\right]$ and the RMS slopes $\left(s_{u}\right.$ and $\left.s_{c}\right)$ should be determined before the peakedness coefficient $(n)$ is determined. It has been well known that the $\sigma_{0}^{\mathrm{dB}}(0)$, as well as $s_{u}$ and $s_{c}$, is expressed as a function of wind speed, so $n$ may also be expressed as a function of wind speed. 


\subsection{Nadir Empirical Model}

Several nadir empirical model functions that relate the Ku-band normal incidence backscatter to the surface wind speeds have been devised using the observations from radar altimeters. For example, Chelton and $\mathrm{McCabe}^{14}$ proposed a logarithmic model function, hereafter referred to as $\mathrm{CM}$, mapping $\sigma_{0}^{\mathrm{dB}}(0)$ to the wind speed at $19.5-\mathrm{m}$ above the sea surface using the coincident data of the SEASAT altimeter and scatterometer. CM is based on the wind speed data between 4 and $14 \mathrm{~m} / \mathrm{s}$ and diverges alarmingly at higher wind speeds. ${ }^{14}$ Young ${ }^{15}$ proposed a linear function valid for wind speeds between 20 and $40 \mathrm{~m} / \mathrm{s}$ by comparing the GEOSAT altimeter crosssections with the predicted wind speeds during satellite overpasses of tropical cyclone. Then, Freilich and Challenor ${ }^{16}$ developed an empirical model function using the GEOSAT altimeter backscatter measurements and buoy wind speeds that is the sum of an exponential and a linear trend and valid for the full range of wind speeds.

Following the above approaches, we assume that the KuPR measured cross-sections at normal incidence depend only on wind speed and that the $\sigma_{0}^{\mathrm{dB}}(0)$ wind speed dependence is modeled as the sum of an exponential (important at low wind speeds) and a linear decrease (dominated at moderate to high wind speeds) as follows:

$$
\sigma_{0}^{\mathrm{dB}}(0)=a+b U_{10}+c \exp \left(d U_{10}\right)
$$

where $U_{10}$ denotes the neutral stability wind at $10 \mathrm{~m}$ and $a, b, c$, and $d$ are parameters that are found by fitting the data. The collocated KuPR-buoy dataset at the nadir incidence angle during the period from January 1 to September 30, 2015, is used to fit the parameters. The wind speed range is binned into $0.2 \mathrm{~m} / \mathrm{s}$ bins, and the collocated $\sigma_{0}^{\mathrm{dB}}(0)$ values are averaged within each bin. The result is plotted as the black points in Fig. 2. Then, the following values are obtained from a nonlinear least squares fit:

$$
a=13.806, \quad b=-0.257, \quad c=4.336, \quad d=-0.524 .
$$

The proposed empirical model function is verified using the remaining 3 months of the collocated dataset. Given the buoy wind speeds, Eq. (9) is used to derive the corresponding radar cross-sections. Then, the resulting radar cross-sections are compared with the GPM KuPR counterparts, clearly indicating that the new model function performs quite well with a bias of $0.06 \mathrm{~dB}$ and a RMSE of $0.94 \mathrm{~dB}$. The comparison is also summarized in the scatter diagram (Fig. 3). It can be seen that the spread becomes larger as the radar backscatter cross-section increases, i.e., as the wind speed decreases. In other words, the error of the empirical model is much larger at weaker wind speeds.

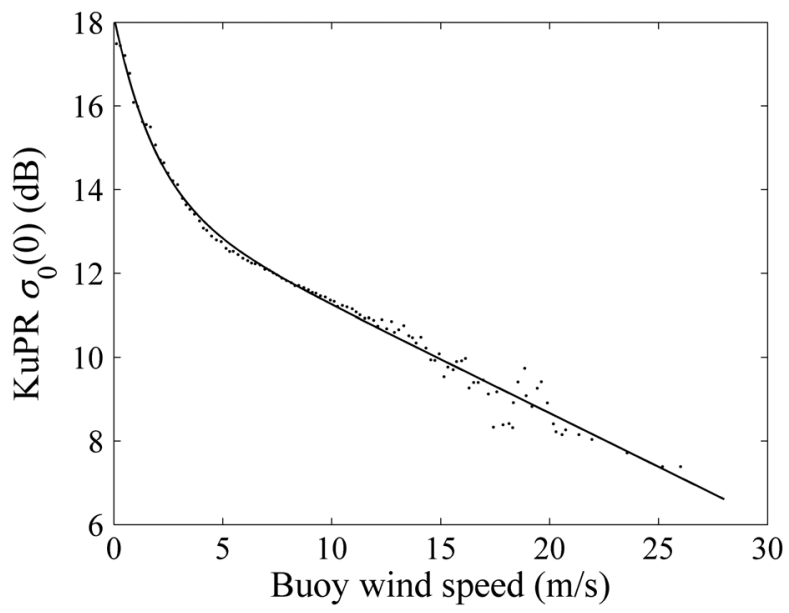

Fig. 2 Relation between the KuPR normal incidence backscatter and buoy wind speed. The black points are the mean values of the KuPR measurements for given wind speed bins. The black line is the proposed empirical model function. 
Yan et al.: Study of sea-surface slope distribution and its effect on radar backscatter...

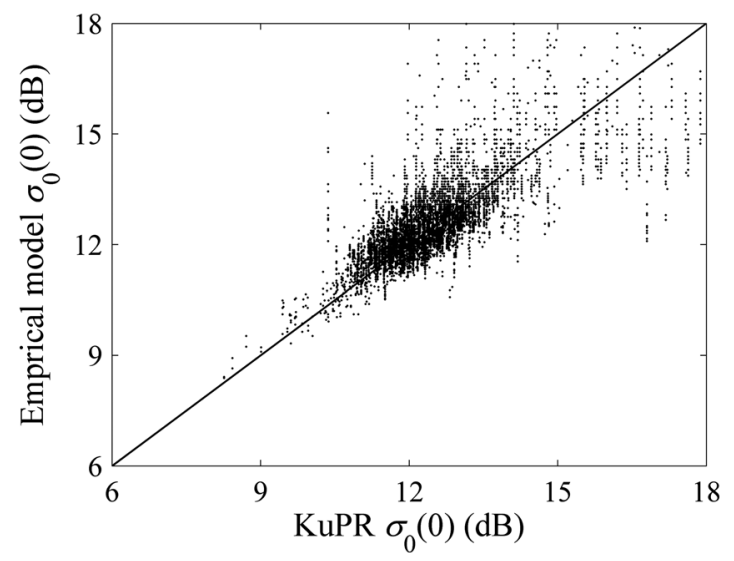

Fig. 3 Scatterplot for the empirical model predictions with respect to the KuPR measurements.

\subsection{Mean Square Slope}

The relationship between the (filtered) mean square slope and surface wind speed has been investigated extensively. There is currently no quantitative agreement in the literature about the wind speed dependence of the (filtered) mean square slope. Cox and Munk ${ }^{5}$ determined the mean square slope components, crosswind, and upwind, under different wind speeds based on the sun glint patterns and suggested that the two components and the total mean square slope vary linearly with wind speed for both clean and slick surfaces. $\mathrm{Wu}^{17}$ reanalyzed the Cox and Munk observations and assumed that the mean square slope varies with the logarithm of the wind speed and the ratio between the crosswind and upwind components varies between 0.6 and 1.0. For the Ku-band, Jackson et al. ${ }^{18}$ determined the filtered mean square slope using airborne Ku-band radar measurements based on the isotropic Gaussian-surface GO scattering model fitting a linear relationship between the filtered mean square slope and wind speed. However, the relationships given by these studies have some limitations. For example, the Cox and Munk results are not applicable for the radar filtered mean square slope, and the results of Jackson et al. ${ }^{18}$ are obtained based on observations over the wind speed range of 7 to $15 \mathrm{~m} / \mathrm{s}$ and the most simplified scattering model and do not contain estimates of the two components.

Here, the dependence of the Ku-band radar filtered mean square slope and its two components on the 10-m wind speed is determined through comparisons of the mean square slopes obtained from the D (Donelan-Banner-Plant), A (Apel), E (Elfouhaily et al.), and H (Hwang) spectra with those obtained by remote sensing techniques. The details of these spectral models have been described by Plant, ${ }^{19}$ Apel, ${ }^{3}$ Elfouhaily et al. ${ }^{20}$ and Hwang, ${ }^{21,22}$ respectively, and will not be repeated here. The $\mathrm{H}$ spectrum is also a function of swell intensity, and $\mathrm{H} 1$ to $\mathrm{H} 4$ denote the $\mathrm{H}$ spectra for 1 to 4 levels of the swell index, with 1 corresponding to mostly wind sea and 4 corresponding to the highest swell influence in the available field data. In this paper, the $\mathrm{H} 1$ and $\mathrm{H} 4$ spectra are used. With the wave spectral models, the filtered mean square slopes are obtained by integrating from zero to an upper bound cutoff wavenumber $k_{c}$ as follows:

$$
\begin{aligned}
s^{2} & =s_{u}^{2}+s_{c}^{2}=\int_{0}^{k_{c}} \int_{-\pi}^{\pi} k^{2} \Psi(k, \phi) k \mathrm{~d} k \\
\mathrm{~d} \phi & =\int_{0}^{k_{c}} k^{2} S(k) \mathrm{d} k, \\
s_{u}^{2} & =\int_{0}^{k_{c}} \int_{-\pi}^{\pi} k^{2} \cos ^{2} \phi \Psi(k, \phi) k \mathrm{~d} k \mathrm{~d} \phi, \\
s_{c}^{2} & =\int_{0}^{k_{c}} \int_{-\pi}^{\pi} k^{2} \sin ^{2} \phi \Psi(k, \phi) k \mathrm{~d} k \mathrm{~d} \phi,
\end{aligned}
$$

where $k$ is the wavenumber magnitude, $\phi$ is its angle with respect to the direction toward which the wind blows, $\Psi(k, \phi)$ is the two-dimensional directional spectrum, and $S(k)$ is the wave height spectrum. The lower limit of the wavelength contributing to the filtered mean square slope is 
Yan et al.: Study of sea-surface slope distribution and its effect on radar backscatter...

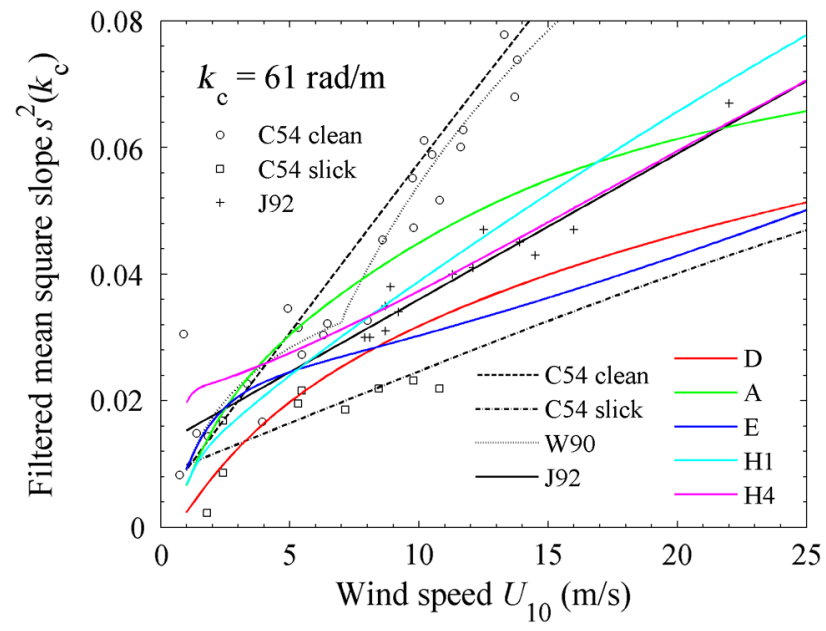

Fig. 4 Filtered mean square slopes obtained by integrating the $D, A, E, H 1$, and $H 4$ spectral functions from zero to the Ku-band cutoff wavenumber, and their comparison with those obtained by remote sensing techniques.

three to six times the radar wavelength; in other words, the cutoff wavenumber $k_{c}$ is a sixth to a third of the radar wavenumber $k_{r}{ }^{18,23}$ Here, the criterion $k_{c}=k_{r} / 4.7$ proposed by Jackson et al. ${ }^{18}$ is adopted. For KuPR measurements, the integration is carried to $k_{c}=61 \mathrm{rad} / \mathrm{m}$.

Figure 4 displays a comparison of the integrated $\mathrm{Ku}$-band filtered mean square slopes from the spectral models mentioned above with the results obtained by remote sensing techniques, including the sun glint data of clean water (black circle) (corresponding to a much larger cutoff wavenumber) and slick water (black square) (corresponding to a smaller cutoff wavenumber) and their relevant linear (black dashed and dash-dotted line) and logarithmic (black dotted line) parameterizations ${ }^{5,17}$ as well as the Ku-band radar data (black plus) and the corresponding fitting line (black solid line). ${ }^{18}$ The mean square slopes integrated from D, A, E, H1, and H4 spectra are almost all smaller than the data for clean water and larger than those for slick water and are around those obtained from Ku-band radar. Using the radar data as reference, the D spectrum underpredicts the filtered mean square slope in all the wind speed ranges. The A spectrum yields good agreement for lower wind speeds (less than approximately $3 \mathrm{~m} / \mathrm{s}$ ) but somewhat overestimates for the moderate to high wind speeds and becomes underestimated at the even higher wind speeds. The E spectrum produces good agreement for low wind speeds (less than approximately $5 \mathrm{~m} / \mathrm{s}$ ) but underestimates for the higher wind speeds. By contrast, the $\mathrm{H}$ spectrum seems to provide an overall better agreement with the $\mathrm{H} 1$ spectrum at relatively low winds (less than approximately $8 \mathrm{~m} / \mathrm{s}$ ) and with the $\mathrm{H} 4$ spectrum at high wind speeds. Thus, the relationship between the filtered mean square slope and 10-m wind speed is determined using the $\mathrm{H} 1$ spectrum for low winds and the $\mathrm{H} 4$ spectrum for high winds. By varying $U_{10}$ from 1 to $25 \mathrm{~m} / \mathrm{s}$ in steps $0.1 \mathrm{~m} / \mathrm{s}$, a dataset of filtered mean square slopes as a function of $U_{10}$ is created. The wind speed dependence is assumed to be linear and the least squares fitting procedure is applied to compute the coefficients. Then, we obtain the following:

$$
s^{2}=0.0026 U_{10}+0.0111, \quad s_{c}^{2} / s_{u}^{2}=0.76 .
$$

\subsection{Peakedness Coefficient Estimation}

From Eqs. (8), (9), and (11), the peakedness coefficient is determined for an arbitrary wind speed. To discuss the wind speed dependence of the peakedness coefficient, values are calculated for the wind speeds from 1 to $25 \mathrm{~m} / \mathrm{s}$ with steps of $0.1 \mathrm{~m} / \mathrm{s}$, and the results are plotted as black dots in Fig. 5. It can be noted that the relationship between the peakedness coefficient and wind speed deviates from linear over the wide range of wind speeds. The least-squares polynomial fitting method is adopted to obtain a simple expression for the relationship. For comparison, the fitting curve is superimposed in Fig. 5 (red curve). The 10th-order polynomials with a RMSE of 0.009 is written as follows: 
Yan et al.: Study of sea-surface slope distribution and its effect on radar backscatter...

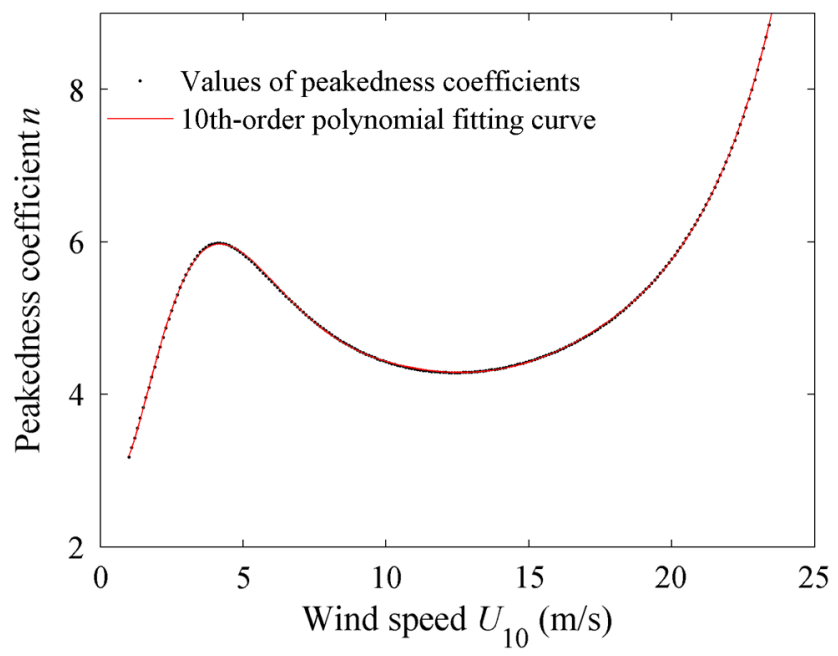

Fig. 5 Peakedness coefficient as a function of wind speed.

Table 1 Coefficients of the polynomial fitting equation.

\begin{tabular}{lccccc}
\hline \hline$P_{0}$ & $P_{1}$ & $P_{2}$ & $P_{3}$ & $P_{4}$ & $P_{5}$ \\
\hline 3.5389 & -2.4564 & 3.0797 & -1.1674 & 0.2270 & -0.0265 \\
$P_{6}$ & $P_{7}$ & $P_{8}$ & $P_{9}$ & $P_{10}$ & \\
0.0020 & $-9.3454 \times 10^{-05}$ & $2.7745 \times 10^{-06}$ & $-4.6799 \times 10^{-08}$ & $3.4292 \times 10^{-10}$ & \\
\hline \hline
\end{tabular}

$$
n=\sum_{i=0}^{10} P_{i} U_{10}^{i} .
$$

The fitting coefficients are listed in Table 1.

All the collocated data of the KuPR backscatter measurements and buoy wind speeds are used to verify the proposed model function. Equation (1), with the inclusions of Eqs. (4), (12), and (11), is used to derive the predictions of the radar cross-sections for the given buoy wind speeds. The predictions are compared with the GPM KuPR counterparts, and the comparison indicates that, when using the KuPR backscatter measurements as a reference, the predictions have a bias of $0.01 \mathrm{~dB}$ and a RMSE of $0.98 \mathrm{~dB}$ for the normal incidence angle and a bias of $-1.35 \mathrm{~dB}$ and a RMSE of $2.74 \mathrm{~dB}$ for all the incidence angles.

\section{Comparison with Measurements}

The performance of the modified Liu, Gaussian, and Gram-Charlier distributions in predicting the radar backscatter at low incidence angles ( $0 \mathrm{deg}$ to $18 \mathrm{deg}$ ) at different sea states is analyzed using the 1 year of collocated, rain-free normalized backscattering cross-section measurements from the GPM KuPR and the in situ winds from the NDBC, TAO, PIRATA, and RAMA moored buoys. For the modified Liu PDF, Eqs. (11) and (12) are used to determine the filtered mean square slope components and peakedness coefficient, respectively. Equation (11) is also used to determine the filtered mean square slope components in the Gaussian PDF. However, for the Gram-Charlier PDF, the mean square slope components, as well as the other five coefficients, are determined by two sets of functions established by Cox and Munk, ${ }^{5}$ one for clean water and the other for slick water.

Table 2 displays the statistical results of the comparisons between the GO model predictions using the modified Liu, Gaussian, and Gram-Charlier (including those for clean water and for 
Yan et al.: Study of sea-surface slope distribution and its effect on radar backscatter...

Table 2 Statistics of the comparisons of the GO model predictions using the modified Liu, Gaussian, Gram-Charlier (including for clean water and for slick water) distributions and the KuPR measurements.

\begin{tabular}{|c|c|c|c|c|c|c|c|c|c|}
\hline \multirow[b]{2}{*}{$\begin{array}{l}\text { Incidence } \\
\text { angle (deg) }\end{array}$} & \multirow[b]{2}{*}{$\begin{array}{l}\text { Wind speed } \\
\text { range }(\mathrm{m} / \mathrm{s})\end{array}$} & \multicolumn{2}{|c|}{ Modified Liu } & \multicolumn{2}{|c|}{ Gaussian } & \multicolumn{2}{|c|}{$\begin{array}{l}\text { Gram-Charlier } \\
\text { clean water }\end{array}$} & \multicolumn{2}{|c|}{$\begin{array}{l}\text { Gram-Charlier } \\
\text { slick water }\end{array}$} \\
\hline & & $\begin{array}{l}\text { Bias } \\
(\mathrm{dB})\end{array}$ & $\begin{array}{l}\text { RMSE } \\
(\mathrm{dB})\end{array}$ & $\begin{array}{l}\text { Bias } \\
\text { (dB) }\end{array}$ & $\begin{array}{l}\text { RMSE } \\
(\mathrm{dB})\end{array}$ & $\begin{array}{l}\text { Bias } \\
(\mathrm{dB})\end{array}$ & $\begin{array}{l}\text { RMSE } \\
(\mathrm{dB})\end{array}$ & $\begin{array}{l}\text { Bias } \\
(\mathrm{dB})\end{array}$ & $\begin{array}{l}\text { RMSE } \\
\text { (dB) }\end{array}$ \\
\hline \multirow[t]{4}{*}{ ALL } & ALL & -1.35 & 2.74 & -1.55 & 2.96 & -2.11 & 16.27 & -2.31 & 4.26 \\
\hline & $<5$ & -0.96 & 3.78 & -1.70 & 4.28 & -3.44 & 27.42 & -2.94 & 5.71 \\
\hline & $5-15$ & -1.56 & 1.97 & -1.47 & 1.89 & -1.39 & 1.78 & -1.97 & 3.22 \\
\hline & $>15$ & -1.02 & 1.49 & -0.78 & 1.21 & -1.67 & 2.07 & -0.64 & 1.71 \\
\hline \multirow[t]{4}{*}{0} & ALL & -0.003 & 1.21 & -0.98 & 1.59 & -1.51 & 2.16 & 1.01 & 1.60 \\
\hline & $<5$ & -0.06 & 1.71 & -1.04 & 2.06 & -0.52 & 2.03 & 0.88 & 1.99 \\
\hline & $5-15$ & 0.03 & 0.83 & -0.94 & 1.27 & -2.02 & 2.22 & 1.07 & 1.36 \\
\hline & $>15$ & 0.09 & 0.93 & -0.99 & 1.36 & -2.74 & 2.90 & 1.18 & 1.51 \\
\hline \multirow[t]{4}{*}{3} & ALL & -0.55 & 1.03 & -1.18 & 1.47 & -1.78 & 2.26 & 0.48 & 1.01 \\
\hline & $<5$ & -0.60 & 1.32 & -1.09 & 1.61 & -0.93 & 2.06 & 0.40 & 1.27 \\
\hline & $5-15$ & -0.53 & 0.84 & -1.22 & 1.39 & -2.22 & 2.35 & 0.53 & 0.84 \\
\hline & $>15$ & -0.25 & 0.83 & -1.15 & 1.40 & -2.86 & 2.97 & 0.84 & 1.17 \\
\hline \multirow[t]{4}{*}{6} & ALL & -1.24 & 1.75 & -1.08 & 1.70 & -1.85 & 4.32 & -0.36 & 1.28 \\
\hline & $<5$ & -1.12 & 2.23 & -0.68 & 2.13 & -1.38 & 6.72 & -0.40 & 1.98 \\
\hline & $5-15$ & -1.30 & 1.44 & -1.30 & 1.43 & -2.09 & 2.21 & -0.35 & 0.68 \\
\hline & $>15$ & -0.68 & 1.00 & -1.09 & 1.31 & -2.65 & 2.75 & -0.40 & 0.83 \\
\hline \multirow[t]{4}{*}{9} & ALL & -1.69 & 2.63 & -0.90 & 2.24 & -1.79 & 9.36 & -1.53 & 2.50 \\
\hline & $<5$ & -1.40 & 3.66 & -0.45 & 3.34 & -2.25 & 15.72 & -1.79 & 3.67 \\
\hline & $5-15$ & -1.85 & 1.98 & -1.15 & 1.32 & -1.54 & 1.73 & -1.40 & 1.55 \\
\hline & $>15$ & -1.03 & 1.27 & -0.80 & 1.06 & -2.09 & 2.21 & -0.12 & 0.67 \\
\hline \multirow[t]{4}{*}{12} & ALL & -1.92 & 3.25 & -1.17 & 2.80 & -2.00 & 16.31 & -3.17 & 2.53 \\
\hline & $<5$ & -1.39 & 4.42 & -1.07 & 4.23 & -3.84 & 27.57 & -3.74 & 5.49 \\
\hline & $5-15$ & -2.23 & 2.39 & -1.24 & 1.53 & -0.99 & 1.33 & -2.86 & 2.99 \\
\hline & $>15$ & -1.34 & 1.58 & -0.52 & 0.94 & -1.36 & 1.54 & -0.91 & 1.12 \\
\hline \multirow[t]{4}{*}{15} & ALL & -1.94 & 3.68 & -2.29 & 3.82 & -2.60 & 22.45 & -5.30 & 6.10 \\
\hline & $<5$ & -1.14 & 5.05 & -2.99 & 5.58 & -6.20 & 37.53 & -6.48 & 7.94 \\
\hline & $5-15$ & -2.40 & 2.64 & -1.92 & 2.33 & -0.60 & 1.22 & -4.66 & 4.78 \\
\hline & $>15$ & -1.62 & 1.95 & -0.51 & 1.19 & -0.65 & 1.04 & -2.05 & 2.21 \\
\hline \multirow[t]{4}{*}{18} & ALL & -1.78 & 3.99 & -4.53 & 6.01 & -3.95 & 35.09 & -8.28 & 9.18 \\
\hline & $<5$ & -0.65 & 5.60 & -6.55 & 8.58 & -10.27 & 58.96 & -10.81 & 12.15 \\
\hline & $5-15$ & -2.39 & 2.74 & -3.43 & 3.96 & -0.48 & 1.36 & -6.91 & 7.05 \\
\hline & $>15$ & -1.92 & 2.31 & -0.98 & 1.67 & 0.03 & 0.86 & -3.55 & 3.66 \\
\hline
\end{tabular}


Yan et al.: Study of sea-surface slope distribution and its effect on radar backscatter...
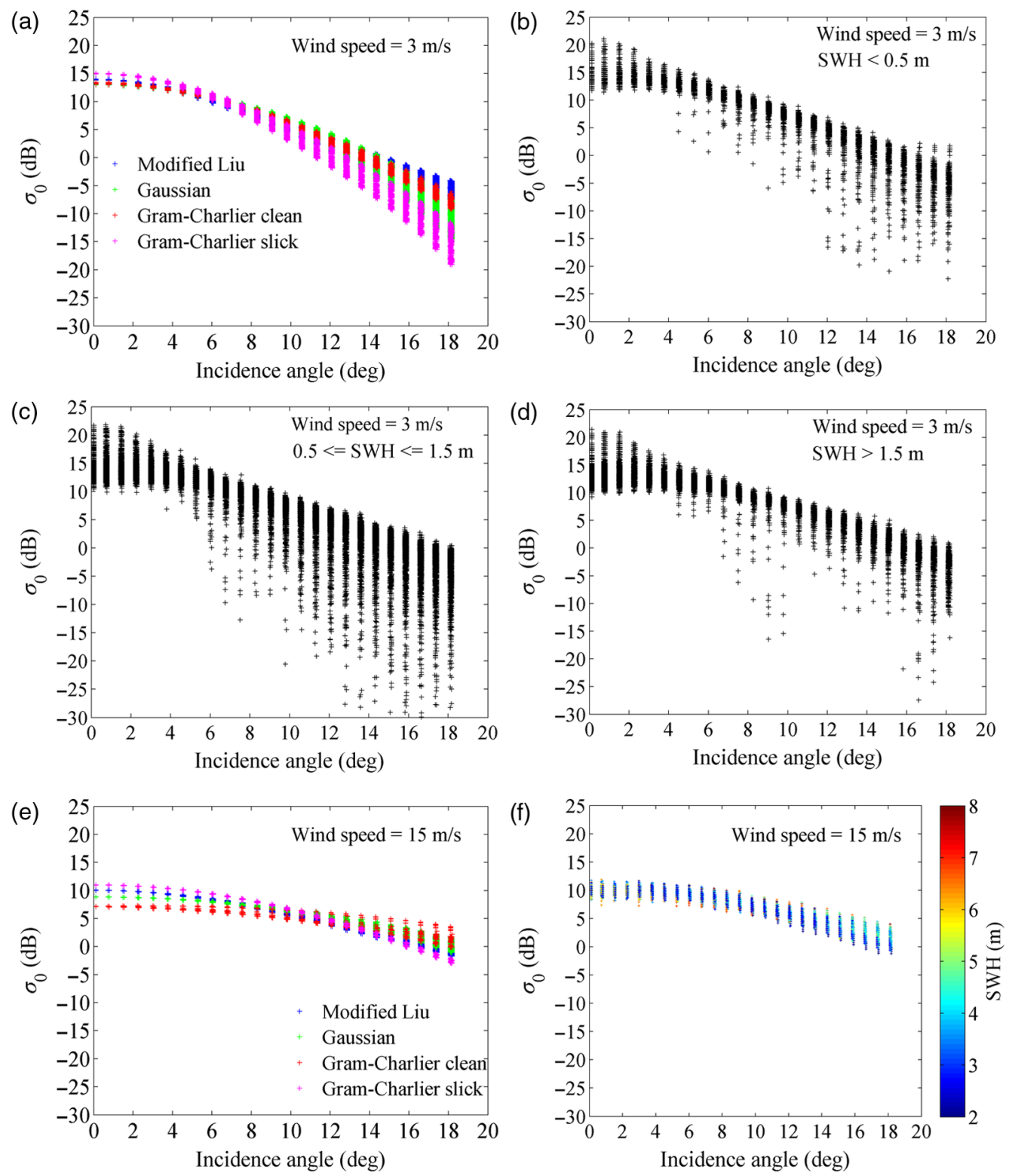

Fig. 6 Scatterplots of the KuPR measurements under different SWH ranges and model predictions versus the incidence angle for the wind speeds of 3 and $15 \mathrm{~m} / \mathrm{s}$. (a) and (e) Model predictions. [(b)-(d), (f)] KuPR measurements.

slick water) distributions and the KuPR measurements for different wind speed ranges and different incidence angles. It can be noted that, overall, the model predictions with the modified Liu distribution agree best with the measurements, followed by those with the Gaussian distribution, then those with the Gram-Charlier distribution for slick water; the predictions with the GramCharlier distribution for clean water show the largest difference from the measurements. The best performance of the modified Liu distribution implies that the model function for the peakedness coefficient determination works well. The fact that the Gram-Charlier distribution, both for clean water and for slick water, performs poorer than the Gaussian distribution may be due to the fact that the Gram-Charlier PDF includes the total or slick filtered and not the radar filtered probability density.

No matter what distribution is used, the biases of the GO model predictions are basically negative, indicating that the model underpredicts the radar backscattering; the RMSEs decrease with increasing wind speeds and decreasing incidence angles, suggesting that the model works better toward higher winds and lower incidence angles. The overall underestimation of the model 
predictions and the bigger errors at the larger incidence angles is probably due to the Bragg scattering component, which is not included in the GO model but becomes more important toward increasing incidence angles, while the bigger errors at lower wind speeds may be due to the effect of the constant presence of background swell on radar backscatter, which is much more obvious at weak winds. ${ }^{13}$ Figure 6 provides evidence of this assertion. It displays the KuPR measurements for different significant wave height $(\mathrm{SWH})$ ranges and model predictions as a function of the incidence angle for wind speeds of 3 and $15 \mathrm{~m} / \mathrm{s}$. It can be seen that, at low wind speed $(3 \mathrm{~m} / \mathrm{s})$, the underestimations of model predictions with respect to the measurements become larger with increasing SWH and higher incidence angles ( $>6 \mathrm{deg})$, where the presence of the swell modifies the local incident angle and, hence, increases the backscattering intensity. By contrast, the underpredictions at high wind speed $(15 \mathrm{~m} / \mathrm{s})$ are much smaller and have no evident relation to the SWH. However, it can be noted that some measurements are much larger than the model predictions at near normal incidence and some are much smaller at high incidences at low wind speeds, which is the opposite of the effect of the presence of background swell. Majurec et al. $^{24}$ assumed that the probable dominant factors in these plots are the great uncertainties in the KuPR measurements and buoy observations during weak winds.

Figure 7 clearly depicts the comparisons of the variations of the KuPR measurments and the model predictions using the modified Liu distribution, Gaussian distribution, and Gram-Charlier distributions for clean and slick water with respect to the buoy wind speeds at different incidence angles. Data are binned into $0.2-\mathrm{m} / \mathrm{s}$ intervals of the buoy wind speed. This binning shows a result similar to that shown in Table 1, demonstrating that, when using the GO model to predict the radar backscatter under different incidence angles, the modified Liu distribution performs best at a near-nadir incidence (near $0 \mathrm{deg}$ ), the Gram-Charlier distribution for slick water is the best when the incidence angle is near $6 \mathrm{deg}$, and the Gaussian distribution is the best when the incidence angle is near $12 \mathrm{deg}$. For an incidence angle near $18 \mathrm{deg}$, the model predictions with the Gram-Charlier distribution for clean water agree very well with the measurements at moderate to high winds, although the error is abnormally large at very low winds $(<2 \mathrm{~m} / \mathrm{s}$ approximately). The good performance of the Gram-Charlier distribution for clean water at high incidence angles and moderate to high winds may be due to the effect of the much larger cutoff wavenumber of the model prediction, which can compensate for the missing Bragg scattering component. The abnormal underestimation at very low winds is probably due to the coefficient determination functions of clean water given by Cox and Munk, ${ }^{5}$ which are not appropriate for extremely low wind speeds. The assertion can be demonstrated indirectly by the fact that, when the functions given by Bréon and Heriot ${ }^{7}$ are used, the large overestimation that occurs at the 0 deg incidence angle becomes smaller; the abnormal underestimation at the 3-deg incidence angle disappears; and under any other incidence angle, the estimation errors become much smaller.

For the lower incidence angles ( $0 \mathrm{deg}$ and $3 \mathrm{deg})$, where the Bragg scattering component is negligible, the overestimations or underestimations of the model predictions with respect to the measurements may be attributed to the uncertainties of the corresponding PDFs when describing the radar detectable slopes. For example, the smaller cutoff wavenumber for slick water than that of Ku-band radar causes the overestimation of the model predictions with the Gram-Charlier PDF for slick water; the larger cutoff wavenumber for clean water causes the underestimation of the model predictions with the Gram-Charlier PDF for clean water over a wide range of wind speed $(>1.5 \mathrm{~m} / \mathrm{s})$. For higher incidence angles $(\geq 6 \mathrm{deg})$, the relative error between the model predictions and the measurements is primarily the result of the sum of the uncertainties of the PDF and ignoring the Bragg scattering component. For example, the good agreement between the model predictions with the Gram-Charlier PDF for slick water and the measurements when the incidence angle is $6 \mathrm{deg}$ is probably because the overestimation, caused by the smaller cutoff wavenumber, is comparable with the underestimation caused by ignoring the Bragg scattering component. In addition, the value of the model prediction error relative to the measurements is rather questionable when the wind speed is very low as the KuPR and buoy measurements are not reliable.

Figure 8 displays the comparison of means of the binned KuPR measurements and model predictions using the modified Liu distribution, Gaussian distribution, and Gram-Charlier distributions for clean and slick water as a function of the incidence angles under different wind 
Yan et al.: Study of sea-surface slope distribution and its effect on radar backscatter...
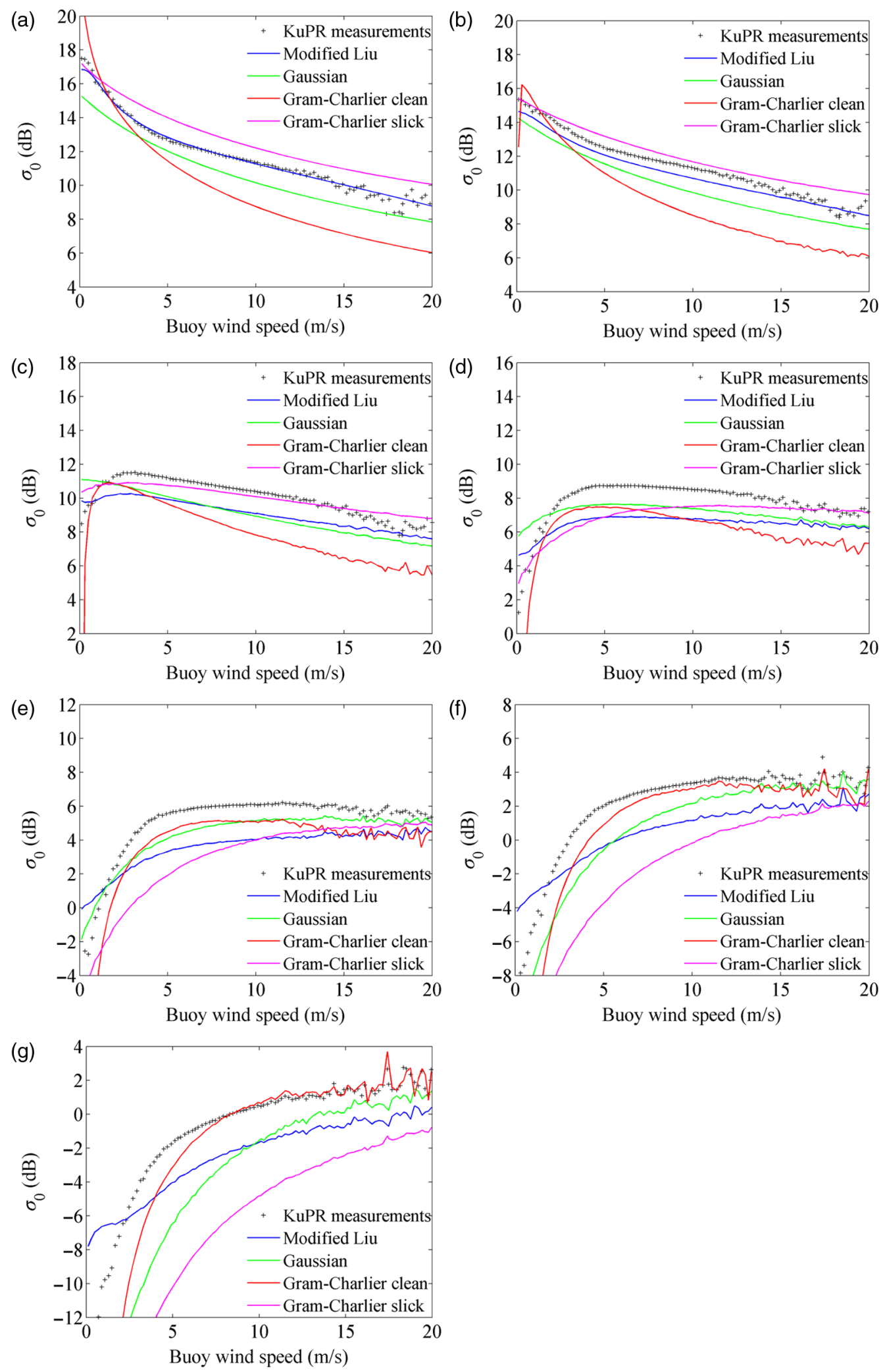

Fig. 7 Mean values of the binned KuPR measurements and model predictions using the modified Liu, Gaussian, and Gram-Charlier (including for clean water and for slick water) distributions as a function of buoy wind speed for the different incidence angles. (a) Incidence angle = $0 \mathrm{deg}$, (b) incidence angle $=3 \mathrm{deg}$, (c) incidence angle $=6 \mathrm{deg}$, (d) incidence angle $=9 \mathrm{deg}$, (e) incidence angle $=12 \mathrm{deg},(\mathrm{f})$ incidence angle $=15 \mathrm{deg}$, and $(\mathrm{g})$ incidence angle $=18 \mathrm{deg}$. 

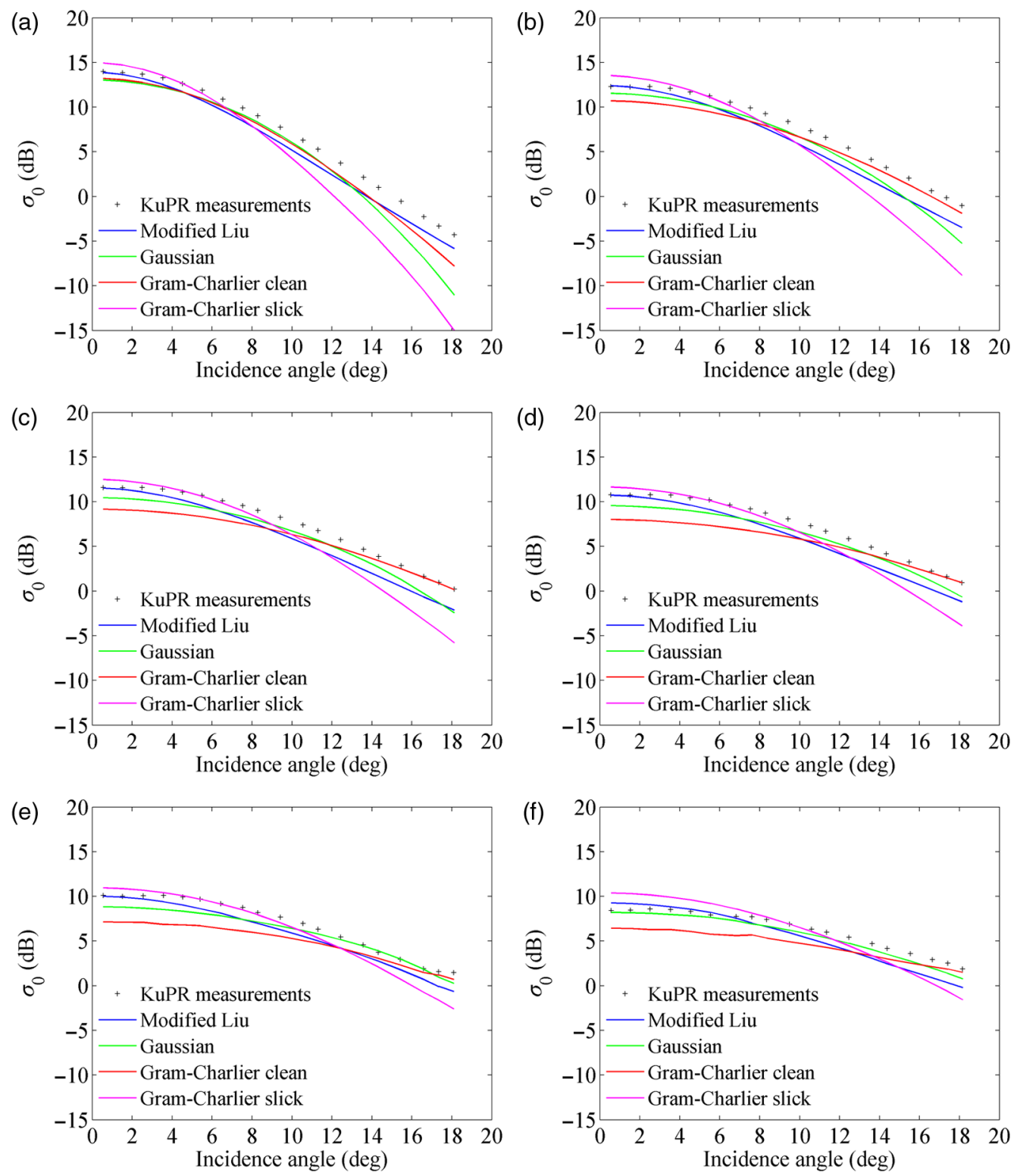

Fig. 8 Mean values of the binned KuPR measurements and model predictions using the modified Liu, Gaussian, and Gram-Charlier (including for clean water and for slick water) distributions as a function of the incidence angle for the different wind speeds. (a) Wind speed $=3 \mathrm{~m} / \mathrm{s}$, (b) wind speed $=6 \mathrm{~m} / \mathrm{s}$, (c) wind speed $=9 \mathrm{~m} / \mathrm{s}$, (d) wind speed $=12 \mathrm{~m} / \mathrm{s}$, (e) wind speed $=$ $15 \mathrm{~m} / \mathrm{s}$, and (f) wind speed $=18 \mathrm{~m} / \mathrm{s}$.

speeds. The data are binned into 1-deg intervals from the incidence angle. For different wind speeds, the pattern of the best-performing distribution with respect to the incidence angle is similar. The modified Liu distribution performs better at relatively low incidence angles. Under lower to middle incidence angles, the Gram-Charlier distribution for slick water performs better, followed by the Gaussian distribution, and the Gram-Charlier distribution for clean water performs better at relatively high incidence angles. The difference between the model predictions with different distributions becomes greater with the wind speed increasing at lower incidence angles and becomes much smaller at higher incidence angles. Generally, the modified Liu distribution performs better than the others at low wind speeds. It also performs quite well under lower incidence angles with moderate to high winds. For the higher incidence angles of the moderate winds, the Gram-Charlier distribution for clean water performs better, and the Gaussian distribution performs well for the higher incidence angles at high winds. 


\section{Conclusion}

The slope distribution of the ocean surface is very important for interpreting radar backscatter measurements. Generally, it is assumed to be Gaussian, but the real sea surface is non-Gaussian. Two non-Gaussian distributions, the Gram-Charlier distribution and the Liu distribution, have been developed by generalizing the Gaussian distribution. In this paper, a method for determining the peakedness coefficient in the Liu distribution is established and the effects of different sea-surface slope distributions (including the modified Liu distribution, the Gaussian distribution, and the Gram-Charlier distribution for clean and slick water) on the GO model predictions of the radar backscatter at low incidence angles ( $0 \mathrm{deg}$ to $18 \mathrm{deg}$ ), and different sea states are analyzed based on the collocated GPM KuPR normalized radar backscattering cross-section measurements and wind measurements from moored buoys.

First, the peakedness coefficient in the Liu distribution is determined using the collocated $\mathrm{KuPR}$ cross-section measurements and buoy wind speeds at the normal incidence angle. It is found that the peakedness coefficient is a nonlinear function of wind speed. The predictions derived from the GO model with the modified Liu PDF (i.e., the Liu PDF with the substitution of the nonlinear function) are compared with the corresponding KuPR measurements, showing a bias of $0.01 \mathrm{~dB}$ and a RMSE of $0.98 \mathrm{~dB}$ for normal incidence, and a bias of $-1.35 \mathrm{~dB}$ and a RMSE of $2.74 \mathrm{~dB}$ for all incidence angles. However, the proposed method to modify the Liu distribution is flawed, e.g., the skewness is ignored, and the mean square slope is determined by referring to the radar data, which are obtained based on the most simplified scattering model (the isotropic, Gaussian surface, GO model). In future work, the skewness will be considered, and the approach to determine the mean square slope will be improved. For example, it can be estimated simultaneously with the peakedness coefficient by fitting Eq. (8) with the empirical model function Eq. (9).

Then, the performance of the modified Liu distribution, the Gaussian distribution, and the Gram-Charlier distribution for clean and slick water is analyzed. Overall, it can be found that the GO model undervalues the radar backscatter mainly by ignoring the Bragg scattering component. On the whole, the modified Liu distribution performs best, followed by the Gaussian distribution, then the Gram-Charlier distribution for slick water. The predictions with the Gram-Charlier distribution for clean water have the largest differences with the measurements as the error is abnormally large at very low winds. For different incidence angles, the modified Liu distribution performs better under relatively low incidence angles and is followed by the Gram-Charlier distribution for slick water and the Gaussian distribution. The Gram-Charlier distribution for clean water performs better at relatively high incidence angles. For different wind speeds, the modified Liu distribution generally performs better than others at low wind speeds, and the Gaussian distribution performs better at high wind speeds. The analysis will provide a basis for the geophysical interpretation of the spaceborne radar at low incidence angles, such as the upcoming Ku-band wave spectrometer, the surface wave investigation and monitoring, onboard the Chinese French Ocean Satellite mission.

\section{Acknowledgments}

This work is supported by the National Key R\&D Program of China under contract No. 2016YFC1401001 and the National Science Foundation of China under contract No. 61501130. The authors would like to thank the Goddard Space Flight Center Distributed Active Archive Center for the straightforward and rapid electronic access to the GPM KuPR data. The authors would also like to thank the American National Oceanic and Atmospheric Administration (NOAA) for providing the buoy wind data. No potential conflict of interest was reported by the authors.

\section{References}

1. D. Barrick, "Rough surface scattering based on the specular point theory," IEEE Trans. Antennas Propag. 16(4), 449-454 (1968).

2. G. R. Valenzuela, "Theories for the interaction of electromagnetic and oceanic waves-a review," Boundary Layer Meteorol. 13(1), 61-85 (1978). 
Yan et al.: Study of sea-surface slope distribution and its effect on radar backscatter...

3. J. R. Apel, "An improved model of the ocean surface wave vector spectrum and its effects on radar backscatter," J. Geophys. Res. 99(C8), 16269-16291 (1994).

4. Y. Liu et al., "The probability density function of ocean surface slopes and its effects on radar backscatter," J. Phys. Oceanogr. 27(5), 782-797 (1997).

5. C. Cox and W. Munk, "Measurement of the roughness of the sea surface from photographs of the sun's glitter," J. Opt. Soc. Am. 44(11), 838-850 (1954).

6. Y. Liu et al., "The mean-square slope of ocean surface waves and its effects on radar backscatter," J. Atmos. Oceanic Technol. 17(8), 1092-1105 (2000).

7. F. M. Bréon and N. Henriot, "Spaceborne observations of ocean glint reflectance and modeling of wave slope distributions," J. Geophys. Res. Oceans 111(C6), 1-10 (2006).

8. D. Evans et al., "Handbook of automated data quality control checks and procedures of the National Data Buoy Center," in NOAA National Data Buoy Center Technical Document, 03-02 (2003).

9. H. P. Freitag et al., "Calibration procedures and instrumental accuracies for ATLAS wind measurements," in NOAA Technical Memorandum OAR PMEL, Vol. 119, p. 20 (2001).

10. H. P. Freitag et al., "ATLAS, T-Flex, BaiLong meteorological sensor comparison test report," in NOAA Technical Memorandum OAR PMEL, Vol. 148, p. 40 (2016).

11. F. T. Ulaby et al., Microwave Radar and Radiometric Remote Sensing, University of Michigan Press, Ann Arbor (2014).

12. J. Wu, "Altimeter wind and wind-stress algorithms-further refinement and validation," J. Atmos. Oceanic Technol. 12(1), 210-215 (1994).

13. P. A. Hwang et al., "A statistical comparison of wind speed, wave height and wave period derived from satellite altimeters and ocean buoys in the Gulf of Mexico region," J. Geophys. Res. 103, 10451-10468 (1998).

14. D. B. Chelton and P. J. McCabe. "A review of satellite altimeter measurement of sea surface wind speed: with a proposed new algorithm," J. Geophys. Res. 90(C3), 4707-4720 (1985).

15. I. R. Young, "An estimate of the Geosat altimeter wind speed algorithm at high wind speeds," J. Geophys. Res. 98(C11), 20275-20285 (1993).

16. M. H. Freilich and P. G. Challenor, "A new approach for determining fully empirical altimeter wind speed model functions," J. Geophys. Res. 99(C12), 25051-25062 (1994).

17. J. Wu, "Mean square slopes of the wind-disturbed water surface, their magnitude, directionality, and composition," Radio Sci. 25(1), 37-48 (1990).

18. F. C. Jackson et al., "Sea surface mean square slope from Ku-band backscatter data," J. Geophys. Res. 97(C7), 11411-11427 (1992).

19. W. J. Plant, "A stochastic, multiscale model of microwave backscatter from the ocean," J. Geophys. Res. 107(C9), 3-1-3-21 (2002).

20. T. Elfouhaily et al., "A unified directional spectrum for long and short wind-driven waves," J. Geophys. Res. 102(C7) 15781-15796 (1997).

21. P. A. Hwang, "Observations of swell influence on ocean surface roughness," J. Geophys. Res. 113(C12), 1-14 (2008).

22. P. A. Hwang et al., "Ocean surface roughness spectrum in high wind condition for microwave backscatter and emission computations," J. Atmos. Oceanic Technol. 30(9), 21682188 (2013).

23. P. A. Hwang et al., "Comparison of composite Bragg theory and quad-polarization radar backscatter from RADARSAT-2: with applications to wave breaking and high wind retrieval," J. Geophys. Res. 115(C8), 246-255 (2010).

24. N. Majurec et al., "Comparison of model predictions with measurements of $\mathrm{Ku}$ and Ka-band near-nadir normalized radar cross sections of the sea surface from the genesis and rapid intensification processes experiment," IEEE Trans. Geosci. Remote Sens. 52(9), 5320-5332 (2014).

Qiushuang Yan is currently a $\mathrm{PhD}$ candidate at Ocean University of China and does her research in Prof. Jie Zhang's group at the First Institute of Oceanography of State Oceanic Administration of China. She received her BS degree from Dalian Maritime University in 2011 and her MS degree in physical oceanography from the First Institute of Oceanography of State Oceanic Administration in 2014. Her research mainly focuses on the mechanism and application of marine radar remote sensing. 
Jie Zhang is a professor at the First Institute of Oceanography of State Oceanic Administration. He received his master's degree from Inner Mongolia University in 1987 and received his $\mathrm{PhD}$ in applied mathematics from Tsinghua University in 1993. His major research interest focuses on remote sensing technology in marine applications.

Chenqing Fan received his bachelor's degree in electronic information engineering in 2006 and his master's degree in signal and information processing technology in 2009 from China University of Petroleum. He is mainly engaged in the application of microwave remote sensing technology in monitoring the changes of ocean dynamic environment.

Jing Wang is a professor at the College of Information and Engineering of Ocean University of China. She received her BS degree from Inner Mongolia Normal University in 1984 and received her PhD in Ocean University of China in 2008. Her research focuses on the application of remote sensing technology in ocean internal wave detection.

Junmin Meng is a professor at the First Institute of Oceanography of State Oceanic Administration. He received his master's degree from Inner Mongolia University in 1999 and received his $\mathrm{PhD}$ in physical oceanography from Ocean University of China in 2002 . He is mainly engaged in the application of synthetic aperture radar remote sensing in marine application research. 